\title{
Medical Treatment with Two Targeted Therapies for an Oncology Patient with Double Primary Cancer: Case Report
}

\author{
Esat NAMAL, ${ }^{1}$ Özgül PAMUKÇU CERCiZ, ${ }^{2}$ Emel GÖKMEN ${ }^{3}$ \\ 'Department of Medical Oncology, İstanbul Bilim University Faculty of Medicine, İstanbul-Turkey \\ ${ }^{2}$ Department of Internal Medicine, Şişli Hamidiye Etfal Training and Research Hospital, İstanbul-Turkey \\ ${ }^{3}$ Department of Internal Medicine, Bağcılar Training and Research Hospital, İstanbul-Turkey
}

\section{Introduction}

The term multiple primary malignant tumor (MPMT) was first used by Billroth in 1889 and was first described in a report by Warren and Gates in 1932 . The criteria developed by Warren and Gates to make a diagnosis of MPMT include the following: each of the tumors must be of different histological type and possibility of metastasis must be excluded.[1] MPMTs are divided into 2 groups, synchrone and metachrone. If a second primary tumor is diagnosed within the first 2 months after the diagnosis of first primary, it is called synchrone. If more than 2 months have elapsed when the second primary is diagnosed, the term metachrone is used.[2] The incidence of MPMTs is reported to be in the range of between $0.4 \%$ and $21.0 \%$ in different countries and various studies.[3-6]

Currently, survival of cancer patients is increasing due to both improvements in treatment modalities and availability of early diagnosis; thus, there will be an increasing need for management of multiple tumors in future. The mechanisms that cause MPMTs are still unclear. While genetic predisposition can play an important role in these cases, such as with hereditary non polyposis coli syndrome, which increases risk for ovarian, endometrial and small intestine cancers as well as colon carcinoma, intensive use of chemotherapy and radiotherapy (RT) might be also associated with certain types of primary cancers. [7-10]

In the last quarter of the 20th century, improvements in hybridoma and recombinant DNA technologies led to use of monoclonal antibodies $(\mathrm{mAb})$ for diagnosis in vitro and ex vivo, and subsequently they have been included in clinical use as a treatment option. Within 30 years, mAb therapy has become part of standard treatment for certain types of cancer.[11]

Targeted cancer therapies are drugs designed to interfere with specific molecules necessary for tumor 
growth and progression. Traditional cytotoxic chemotherapies usually kill rapidly dividing cells in the body by interfering with cell division. A primary goal of targeted therapies is to fight cancer cells with more precision and potentially fewer side effects.[12]

Many different targeted therapies have been approved for use in cancer treatment. These include hormone therapies, signal transduction inhibitors, gene expression modulators, apoptosis inducers, angiogenesis inhibitors, immunotherapies, and toxin delivery molecules.[13]

In terms of studies observing treatment of MPMTs, Zeng et al. investigated hepatocellular carcinoma (HCC) tumors accompanied by non-HCC primary tumors and reported that it was important to treat both the synchrone and metachrone tumors, while also pointing out need for additional studies to support their observations.[14]

Search of literature revealed no reports in which more than one targeted therapy was administered for multiple primary tumors. Described in the present case is multiple primary carcinoma - primary lung cancer and ductal invasive breast carcinoma - along with liver metastasis for which targeted therapies for both lung and breast cancer were administered.

\section{Case Report}

A 70-year-old woman presented at internal medicine outpatient clinic with complaints of fever, weight loss, and breathlessness. Following lung X-ray that showed suspected tumor signs, computed tomography (CT) was performed. Tumors were found in multiple lymph nodes - max. 10x5 mm - in prevascular and aorticopulmonary sites, bilateral hilus and precarinal sites, and a centrally located mass with irregular margins, 38 x 25 $\mathrm{mm}$ in size, was found at the lower lobe of right lung. Diagnosis of adenocarcinoma was made with bronchoscopic biopsy. Mutation of activin receptor-like kinase 1 gene (ALK1) was negative while epidermal growth factor receptor (EGFR) was positive. Positron emission tomography (PET) was performed for staging. Apart from lymphadenopathy seen in CT, hypermetabolic mass lesion suggesting metastasis at segment 7 of the liver, hypermetabolic lymphadenopathy at hepatic hilus, and focal hypermetabolic lesion $10 \mathrm{~mm}$ in diameter in upper middle quadrant of right breast were found, suggesting secondary primary. In addition, bone metastasis located at first thoracic vertebra, right iliac bone and right ischium was detected. Tru cut biopsy of the breast showed invasive ductal carcinoma.
Immunohistochemically estrogen and progesterone receptors were found to be $>\% 90$ positive, and c-erbB2 (HER2/neu) was found to be strongly positive. As the patient was evaluated as multiple primary, biopsies of liver metastasis to determine primary was planned; however, approval of the patient could not be obtained for biopsy. Before announcement of results of immunohistochemical and genetic testing, 4 cycles of chemotherapy with paclitaxel $250 \mathrm{mg}$, carboplatin $600 \mathrm{mg}$ and zoledronat $4 \mathrm{mg}$ were administered every 3 weeks. After chemotherapy, a partial metabolic regression in lesion located in lower lobe - posterobasal segment of left lung - a complete regression in lymphadenopathies located in right lower paratracheal site, and complete regression of mass in right breast was obtained. Furthermore, metastatic mass lesion observed initially in segment 7 of liver was not reported. Since EGFR was positive in lung adenocarcinoma and c-erb was positive in breast adenocarcinoma immunohistochemically, maintenance treatment was as follows: erlotinib $150 \mathrm{mg} /$ day, letrozole $2.5 \mathrm{mg}$ /day, trastuzumab $520 \mathrm{mg}$ loading dose followed by $420 \mathrm{mg}$ was continued for 12 cycles every 3 weeks. At ninth month of treatment, patient was still in follow-up, with assessment of stable disease according to the Response Evaluation Criteria In Solid Tumors (RECIST) version 1.1.[15]

\section{Discussion}

While there is no data regarding administration of both erlotinib and trastuzumab for patients with double primary lung and breast cancers in literature, there are a few case series of administering c-erb targeted treatments to HER2 positive patients as phase I/II studies. [16] These drugs were well tolerated in our study, and there no drug interactions were found in databases. Administration of these 2 targeted therapy modalities in patients with HER2 positive breast cancer was still in progress at the time of present case and there is no data regarding use of more than one targeted therapy in patients with metastatic multiple primary cancer.

In a study investigating outcomes of synchrone non-small cell lung cancers, it was shown that best outcomes can be obtained with more than 1 targeted therapy if tumor is resectable; however, these synchrone tumors were only tumors originating in the lung.[17] In the present case, patient was evaluated as non-resectable due to liver metastasis, primary nature of which could not be clarified because patient did not give approval for biopsy.

In literature, outcomes of multiple primary cancers 
that were accompanied by lung cancer have been investigated.[18-20] In a study conducted by $\mathrm{Li}$ et al., it was reported that the most common cancers to exist concurrently with lung cancer were of digestive system; breast cancer was included in the group of "others," which compromised 9.7\% of total.[21] However, another study reported that concurrence of lung and breast cancer made up highest percentage for both metachrone and synchrone groups of patients with multiple primary tumors.[22]

Most side effects of targeted therapies are directly related to the specific molecular target in normal tissue inhibited or modulated by the specific drug. As present case involves administration of 2 targeted therapies, potential side effects of both were considered. The most commonly observed side effect with EGFR inhibitors is an acneiform eruption, also called acne-like rash or folliculitis, which occurs in $50 \%$ to $100 \%$ of patients. [23-25] Acneiform eruption is less frequent with erlotinib compared to cetuximab or panitumumab.[26]

Post-chemotherapy diarrhea is the result of extensive crypt damage in the small bowel and colon, resulting in excess fluid in the bowel lumen. Exact pathophysiology of anti-EGFR agent-related diarrhea remains unclear. EGF is involved in the maintenance of mucosal integrity and is also a potent mitogen of the gastric epithelium; it stimulates mucin production and enhances prostaglandin synthesis. $[27,28]$ In the present case, neither skin toxicity nor intestinal toxicity was experienced with erlotinib treatment.

Trastuzumab was humanized from the original $\mathrm{mAb}$, thereby allowing chronic administration in humans without development of human anti-mouse antibodies response, which is characterized by anaphylactic response or other immune reactions and rapid drug clearance.[29] Approximately 40\% of patients receiving trastuzumab experience some degree of infusion-related symptoms such as a flu-like syndrome that includes fever and chills, mainly during the first infusion. Other common reactions include tumor site pain, shortness of breath, muscle weakness, cutaneous rash, diarrhea, and headache. Trastuzumab is administered intravenously over 90 minutes for the first infusion with a primary line of saline solution. If first infusion is well tolerated, subsequent doses can be administered over 30 minutes.[26] One major concern is cardiac toxicity related to trastuzumab administration. Cardiac dysfunction was specifically defined as follows: cardiomyopathy characterized by decrease in left ventricular ejection fraction (LVEF), either global or more severe in the septum; symptoms of congestive heart failure
(CHF); associated signs of CHF; decline in LVEF of at least $5 \%$ to $<55 \%$ with signs and symptoms of CHF; or a decline of $10 \%$ to $<55 \%$ without signs or symptoms of CHF. Trastuzumab is associated with increased risk of cardiac dysfunction, which is greatest in patients receiving concurrent anthracyclines.[30]

In the present case, echocardiogram was performed at initiation of therapy. LVEF was initially $60 \%$ and there was no decrease seen during or after treatment.

It is clear that resectability and adverse effects can create difficulties in treatment of multiple primary tumors. However, targeted therapies are developing rapidly and there are promising reports that these modalities will be widely used in the near future. Since there is no definitive modality for treating patients with multiple primary tumors at this time, it is the suggestion of the authors that treatments targeting the same pathways to hit the tumor could be a promising choice of treatment. In the present case, we obtained a better survival than expected with 2 targeted therapies. The result must definitely be supported by further studies in terms of drug interactions and side effects.

\section{Conflict of interest: None declared.}

\section{References}

1. Warren S, Gates O. Multiple primary malignant tumors: A survey of the literature and a statistical study. Am J Cancer 1932;16:1358-414.

2. Hayat MJ, Howlader N, Reichman ME, Edwards BK. Cancer statistics, trends, and multiple primary cancer analyses from the Surveillance, Epidemiology, and End Results (SEER) Program. Oncologist 2007;12(1):20-37.

3. Liu FS, Qin DX, Wang QL. The clinical pathology analysis of 172 cases of multiple primary malignant tumors. Chin J Oncol 1979;1(2):113.

4. Babacan NA, Aksoy S, Cetin B, Ozdemir NY, Benekli $\mathrm{M}$, Uyeturk U, et al. Multiple primary malignant neoplasms: multi-center results from Turkey. J BUON 2012;17(4):770-5.

5. Frödin JE, Ericsson J, Barlow L. Multiple primary malignant tumors in a national cancer registry-reliability of reporting. Acta Oncol 1997;36(5):465-9.

6. Macdonald EJ. Occurrence of multiple primary cancers in a population of 200,000. Acta Unio Int Contra Cancrum 1960;16:1702-10.

7. Chaturvedi AK, Engels EA, Gilbert ES, Chen BE, Storm H, Lynch CF, et al. Second cancers among 104,760 survivors of cervical cancer: evaluation of long-term risk. J Natl Cancer Inst 2007;99(21):1634-43. 
8. Noronha V, Berliner N, Ballen KK, Lacy J, Kracher J, Baehring J, et al. Treatment-related myelodysplasia/ AML in a patient with a history of breast cancer and an oligodendroglioma treated with temozolomide: case study and review of the literature. Neuro Oncol 2006;8(3):280-3.

9. Merrouche Y, Mugneret F, Cahn JY. Secondary acute promyelocytic leukemia following irinotecan and oxaliplatin for advanced colon cancer. Ann Oncol 2006;17(6):1025-6.

10. Erikci AA, Ozturk A, Tekgunduz E, Sayan O. Acute myeloid leukemia complicating multiple myeloma: a case successfully treated with etoposide, thioguanine, and cytarabine. Clin Lymphoma Myeloma 2009;9(4):14-5.

11. Demirelli FH. Monoclonal antibodies for targeted cancer therapy. ANKEM Derg 2005;19(Suppl 2):123-5.

12. Abramson, R. Overview of targeted therapies for cancer. My Cancer Genome 2016.

13. Badhe KP, Phadtare D, Saudagar R. Review on approaches in anticancer drug therapy. IJUPBS 2015;4(4).

14.Zeng QA, Qiu J, Zou R, Li Y, Li S, Li B, et al. Clinical features and outcome of multiple primary malignancies involving hepatocellular carcinoma: a long-term follow-up study. BMC Cancer 2012;12:148.

15. Eisenhauer EA, Therasse P, Bogaerts J, Schwartz LH, Sargent D, Ford R, et al. New response evaluation criteria in solid tumours: revised RECIST guideline (version 1.1). Eur J Cancer 2009;45(2):228-47.

16. Britten CD, Finn RS, Bosserman LD, Wong SG, Press MF, Malik M, et al. A phase I/II trial of trastuzumab plus erlotinib in metastatic HER2-positive breast cancer: a dual ErbB targeted approach. Clin Breast Cancer 2009;9(1):16-22.

17. Jung EJ, Lee JH, Jeon K, Koh WJ, Suh GY, Chung MP, et al. Treatment outcomes for patients with synchronous multiple primary non-small cell lung cancer. Lung Cancer 2011;73(2):237-42.

18. Liu YY, Chen YM, Yen SH, Tsai CM, Perng RP. Multiple primary malignancies involving lung cancerclinical characteristics and prognosis. Lung Cancer 2002;35(2):189-94.

19. Hatta T, Matsuda S, Kurisu S, Ohyabu H, Koyama T, Kita $Y$, et al. Nineteen multiple primary cancer cases of 100 patients undergoing lung cancer resection. [Article in Japanese] Kyobu Geka 1995;48(13):1101-4. [Abstract]

20. Yilmaz A, Ertugrul M, Yagci Tuncer L, Sulu E,
Damadoglu E. Multiple primary malignancies involving lung: an analysis of 40 cases. Ups J Med Sci 2008;113(2):193-200.

21. Li F, Zhong WZ, Niu FY, Zhao N, Yang JJ, Yan HH, et al. Multiple primary malignancies involving lung cancer. BMC Cancer 2015;15:696.

22. Aydiner A, Karadeniz A, Uygun K, Tas S, Tas F, Disci R, et al. Multiple primary neoplasms at a single institution: differences between synchronous and metachronous neoplasms. Am J Clin Oncol 2000;23(4):364-70.

23. Cunningham D, Humblet Y, Siena S, Khayat D, Bleiberg H, Santoro A, et al. Cetuximab monotherapy and cetuximab plus irinotecan in irinotecan-refractory metastatic colorectal cancer. N Engl J Med 2004;351(4):337-45.

24. Ranson M, Hammond LA, Ferry D, Kris M, Tullo A, Murray PI, et al. ZD1839, a selective oral epidermal growth factor receptor-tyrosine kinase inhibitor, is well tolerated and active in patients with solid, malignant tumors: results of a phase I trial. J Clin Oncol 2002;20(9):2240-50.

25. Soulieres D, Senzer NN, Vokes EE, Hidalgo M, Agarwala SS, Siu LL. Multicenter phase II study of erlotinib, an oral epidermal growth factor receptor tyrosine kinase inhibitor, in patients with recurrent or metastatic squamous cell cancer of the head and neck. J Clin Oncol 2004;22(1):77-85.

26. Widakowich C, de Castro G Jr, de Azambuja E, Dinh P, Awada A. Review: side effects of approved molecular targeted therapies in solid cancers. Oncologist 2007;12(12):1443-55.

27. Matsuura M, Okazaki K, Nishio A, Nakase H, Tamaki $\mathrm{H}$, Uchida $\mathrm{K}$, et al. Therapeutic effects of rectal administration of basic fibroblast growth factor on experimental murine colitis. Gastroenterology 2005;128(4):975-86.

28. Playford RJ, Ghosh S, Mahmood A. Growth factors and trefoil peptides in gastrointestinal health and disease. Curr Opin Pharmacol 2004;4(6):567-71.

29. Carter P, Presta L, Gorman CM, Ridgway JB, Henner D, Wong WL, et al. Humanization of an anti-p185HER2 antibody for human cancer therapy. Proc Natl Acad Sci U S A 1992;89:4285-9.

30. Seidman A, Hudis C, Pierri MK, Shak S, Paton V, Ashby $\mathrm{M}$, et al. Cardiac dysfunction in the trastuzumab clinical trials experience. J Clin Oncol 2002;20(5):1215-21. 